\title{
Genetic analysis of the Vitamin D receptor start codon polymorphism (FokI) in cervical vertebra and lumbar spine pathologies: a meta-analysis
}

\author{
Xinyu $\mathrm{Hu}^{1,2}$, Min Liu ${ }^{1}$, Yanjun $\mathrm{Ni}^{2}$ and Guolong Zhang ${ }^{2}$ \\ ${ }^{1}$ Department of Scientific Research, Jiading District Central Hospital Affiliated Shanghai University of Medicine and Health \\ Sciences, Shanghai, China \\ ${ }^{2}$ The Former Dalian Sanatorium of Shenyang Military Region, Dalian, Liaoning, China \\ Correspondence to: Min Liu, email: xiaoshiliu20609@sina.com \\ Guolong Zhang, email: dragoncheung@icloud.com
}

Keywords: Vitamin D receptor (VDR), FokI polymorphism, spinal diseases, cervical vertebra and lumbar spine pathologies Received: June 06, 2017

Accepted: August 08, 2017

Published: August 21, 2017

Copyright: $\mathrm{Hu}$ et al. This is an open-access article distributed under the terms of the Creative Commons Attribution License 3.0 (CC BY 3.0), which permits unrestricted use, distribution, and reproduction in any medium, provided the original author and source are credited.

\section{ABSTRACT}

Background: Vitamin D receptor (VDR) FokI polymorphism has been reported to influence the risk of spinal diseases. However, several studies suggest inconsistent results. Therefore, we performed this analysis to reveal the accurate relationship between VDR FokI polymorphism and spinal diseases.

Materials and Methods: 8 articles accord with the strict inclusion and exclusion criteria. 1116 cases and 1263 controls are entered into this analysis. The pooled odds ratios (ORs) and $95 \%$ confidence intervals (CI) are calculated to evaluate the association between VDR gene polymorphism and spinal diseases.

Result: The results suggest that allele $F$ is a risk factor for spinal diseases and the difference is significant ( $F$ vs. $f: O R=1.151,95 \%$ CI, 1.020-1.300). For the genotype analysis of VDR FokI, no statistical differences exist in the models of heterozygote comparison (Ff vs. ff), homozygote comparison (FF vs. ff) and dominant model (FF + Ff vs. ff) $(p>0.05)$. However, in recessive model (FF vs. Ff + ff $)$, there is a significant association between VDR polymorphism and spinal diseases (OR $=1.209,95 \% \mathrm{CI}$, 1.017-1.436). In subgroup analysis, the results show that allele $F$ is a risk factor for spinal diseases in each estimation. In hospital-based subgroup, the significant differences exist in FF vs. ff and FF vs. Ff + FF models. In degenerative spine diseases group, the results are consistent with that of overall studies.

Conclusions: According to results of this meta-analysis, allele $F$ is associated with the increased risk of spinal diseases. FF genotype may contribute to the susceptibility of spinal diseases. Therefore, VDR FokI polymorphism is related with spinal diseases.

\section{INTRODUCTION}

Cervical spondylosis and lumbar spine pathologies are common diseases of musculoskeletal disorder. Neck and low back pain are the typical symptoms $[1,2]$. The chronic hazards of these diseases are serious threat to human health. The previous researches show that compressive forces on vertebral endplates, obesity, bone mineral density and occupational factors are associated with the disease of vertebral body lesions, such as lumbar spine pathologies, lumbar disc herniation, degeneration of lumbar disc and spinal stenosis, cervical spondylotic myelopathy, ligament disease and so on [3-5]. While recent investigations suggest that genetic factors are also important causes of cervical vertebra and lumbar spine pathologies [6-8]. Vitamin D receptor (VDR) gene is the thoroughly researched candidate gene, which is connected with vertebral diseases. The VDR gene is located on chromosome 12q13.11 and is consisted of 11 exons [9]. There are numerous single nucleotide polymorphisms (SNPs) of VDR gene. FokI (rs2228570), 
BsmI (rs1544410), ApaI (rs7975232) and TaqI (rs731236) are the most frequently investigated SNPs of VDR gene [10]. The FokI polymorphism is a $\mathrm{C} / \mathrm{T}$ single nucleotide transformation on chromosome 12 , which is considered to be one of the most crucial genetic factors affecting the incidence of vertebral diseases. This change induces the transformation from ATG to $\mathrm{ACG}$, resulting in the alternation of protein product [11]. Although the relationship between vitamin D receptor (VDR) gene and spine pathologies is studied systematically, the research results are inconsistent.

Therefore, it is necessary to update the research results of the association between VDR FokI polymorphism and vertebral diseases, which could provide further evidence to discover the real relationship between them. In this study, a meta-analysis is performed to assess the available articles.

\section{RESULTS}

\section{Search strategy and characteristics of eligible articles}

The complete searching procedure is shown in Figure 1. 8 eligible studies including 7 hospital-based studies [17-23] and 1 population-based study [24] are collected in this meta-analysis on the basis of the inclusion criteria. The general information of the eligible articles including the first author, publication year, original country, disease category, control source, diagnostic method and genotyping technique are collected by two independent investigators. The characteristics of included studies are shown in Table 1. 1116 cases and 1263 controls from these articles are employed for the analysis of VDR FokI polymorphism. In addition, numbers of genotype FF, $\mathrm{Ff}$ and $\mathrm{ff}$ in two groups, the total case numbers and control numbers of included studies are collected to calculate the pooled odds ratio (OR) and the VDR FokI genotype distribution information are exhibited in Table 2.

\section{Publication bias}

In this study, publication bias is recognized by Begg's test. The results indicate that there is no notable evidence of publication bias in these comparison models, including the allelic model (F vs. f, Begg's test: $p=1.000$ ), homozygote comparison model (FF vs. ff, Begg's test: $p=0.266$ ) and recessive model (FF vs. Ff + ff, Begg's test: $p=0.902$ ) (Figure 2A-2C). However, significant publication biases are detected in the model of heterozygote comparison (Ff vs. ff, Begg's test: $p=0.019$ ) and dominant model (FF + Ff vs. ff, Begg's test: $p=0.019)$

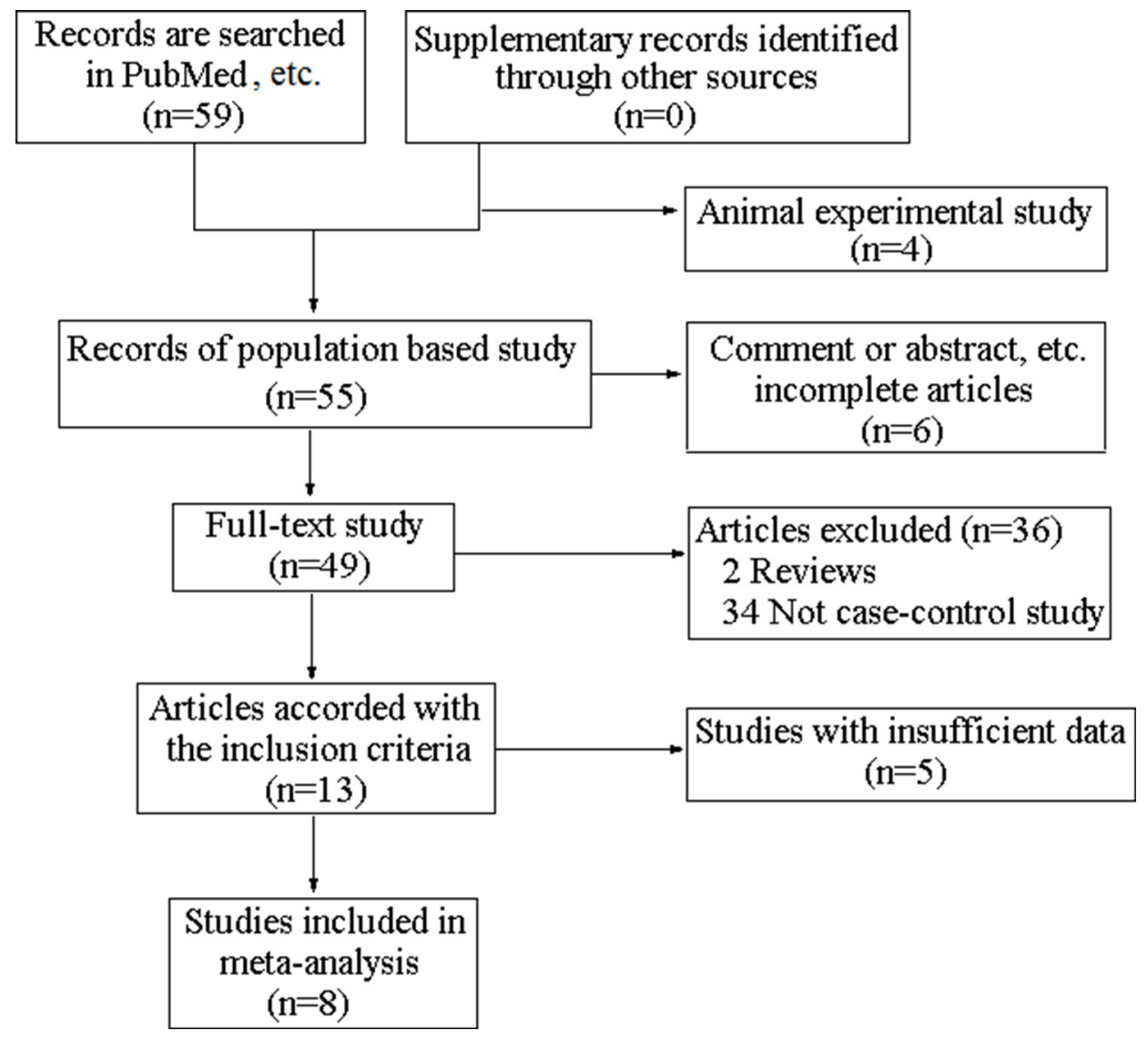

Figure 1: The flowchart of studies identification. 
Table 1: Characteristics of eligible articles

\begin{tabular}{|c|c|c|c|c|c|c|c|}
\hline $\begin{array}{l}\text { First } \\
\text { author }\end{array}$ & $\begin{array}{c}\text { Publication } \\
\text { year }\end{array}$ & Country & Disease & $\begin{array}{l}\text { Diagnostic } \\
\text { method }\end{array}$ & $\begin{array}{l}\text { Genotyping } \\
\text { method }\end{array}$ & $\begin{array}{l}\text { Control } \\
\text { source }\end{array}$ & Score \\
\hline Sansoni V & 2016 & Italy & Lumbar disc herniation & MRI & PCR-RFLP & HB & 9 \\
\hline Colombini A & 2015 & Italy & Lumbar spine pathologies & MRI & PCR-RFLP & $\mathrm{HB}$ & 8 \\
\hline Nikolova S & 2015 & Bulgaria & Idiopathic scoliosis & Radiodiagnosis & PCR-RFLP & $\mathrm{HB}$ & 8 \\
\hline Vieira LA & 2014 & Brazil & $\begin{array}{l}\text { Intervertebral disc } \\
\text { degeneration }\end{array}$ & MRI & PCR-RFLP & $\mathrm{HB}$ & 8 \\
\hline Colombini A & 2014 & Italy & Lumbar spine pathologies & MRI & PCR-RFLP & $\mathrm{HB}$ & 8 \\
\hline Wang ZC & 2010 & China & $\begin{array}{l}\text { Cervical spondylotic } \\
\text { myelopathy }\end{array}$ & MRI & PCR-RFLP & $\mathrm{HB}$ & 9 \\
\hline Kobashi G & 2008 & Japan & $\begin{array}{l}\text { Ossification of the posterior } \\
\text { longitudinal ligament }\end{array}$ & Radiography & PCR-RFLP & $\mathrm{HB}$ & 9 \\
\hline $\begin{array}{l}\text { Noponen } \\
\text {-Hietala N }\end{array}$ & 2003 & Finland & $\begin{array}{l}\text { Degenerative lumbar } \\
\text { spinal stenosis }\end{array}$ & MRI & PCR-RFLP & $\mathrm{PB}$ & 5 \\
\hline
\end{tabular}

PCR-RFLP: polymerase chain reaction-restriction fragment length polymorphism

HB: hospital-based PB: population-based;

Table 2: VDR FokI genotype and allele distribution of eligible studies

\begin{tabular}{|c|c|c|c|c|c|c|c|c|c|c|c|}
\hline \multirow{2}{*}{ First author } & \multicolumn{3}{|c|}{ Case } & \multicolumn{3}{|c|}{ Control } & \multicolumn{2}{|c|}{ Case } & \multicolumn{2}{|c|}{ Control } & \multirow{2}{*}{$\begin{array}{l}P \text { value of } \mathrm{HWE} \\
\text { in control group }\end{array}$} \\
\hline & FF & Ff & ff & FF & Ff & ff & $\mathbf{F}$ & f & $\mathbf{F}$ & f & \\
\hline Sansoni V & 53 & 44 & 13 & 44 & 51 & 15 & 150 & 70 & 139 & 81 & 0.971 \\
\hline Colombini A & 117 & 120 & 30 & 101 & 117 & 36 & 354 & 180 & 319 & 189 & 0.821 \\
\hline Nikolova S & 56 & 43 & 6 & 108 & 92 & 10 & 155 & 55 & 308 & 112 & 0.082 \\
\hline Vieira LA & 17 & 50 & 54 & 10 & 46 & 75 & 84 & 158 & 66 & 196 & 0.434 \\
\hline Colombini A & 117 & 120 & 30 & 89 & 99 & 32 & 354 & 180 & 277 & 163 & 0.601 \\
\hline Wang ZC & 46 & 77 & 31 & 50 & 76 & 30 & 169 & 139 & 176 & 136 & 0.907 \\
\hline Kobashi G & 31 & 21 & 11 & 37 & 70 & 19 & 83 & 43 & 144 & 108 & 0.132 \\
\hline Noponen-Hietala $\mathrm{N}$ & 11 & 12 & 6 & 25 & 26 & 5 & 34 & 24 & 76 & 36 & 0.630 \\
\hline
\end{tabular}

HWE: Hardy-Weinberg equilibrium

(Figure 3A, 3B). "Trim and fill" method is performed to incorporate the hypothetical researches to recalculate the pooled risk assessment. After adjustment, the publication bias did not exist in the two models any more (Figure 3C, 3D). Therefore, there is no obvious publication bias in all comparison models.

\section{Meta-analysis}

\section{Contrastive analysis of allele in all eligible studies}

The results of VDR FokI allelic comparison (F vs. f) in case and control groups are listed in Figure 4. In fixed-effect model, the heterogeneity of subgroups have no statistical significance (chi-squared $=8.33, \mathrm{I}^{2}=16.0 \%$, $p=0.304$ ). The pooled results indicate that allele $\mathrm{F}$ is a risk factor for vertebral diseases compared with allele f (F vs. f: $\mathrm{OR}=1.151,95 \% \mathrm{CI}, 1.020-1.300)$ and the difference is statistically significant $(Z=2.27, p=0.023)$.

\section{Gene polymorphism analysis}

In order to reveal the relationship between FokI polymorphism and spinal diseases, subgroup analysis is divided into 4 parts: homozygote comparison (FF vs. ff), heterozygote comparison (Ff vs. ff), dominant model $(\mathrm{FF}+\mathrm{Ff}$ vs. ff) and recessive model (FF vs. Ff $+\mathrm{ff})$. The results of these subgroups are described in Figures 5-8. As shown in Figure 5, the results suggest that FF genotype is not a risk factor for spinal diseases. In fixed-effect model, the heterogeneity chi-squared value is 7.14 and there is no significant difference $\left(\mathrm{I}^{2}=2.0 \%, p=0.414\right)$. The pooled OR is $1.261(95 \% \mathrm{CI}, 0.969-1.641)$ and there is no significant difference in $Z$ test $(Z=1.73, p=0.084)$.

In Figure 6, it is shown that there is no difference in pathogenesis of spinal diseases between the $\mathrm{Ff}$ and $\mathrm{ff}$ carriers. The chi-squared test of heterogeneity indicate that there is no difference in subgroups on the basis of fixedeffect results (chi-squared $=7.43, \mathrm{I}^{2}=5.8 \%, p=0.385$ ). 

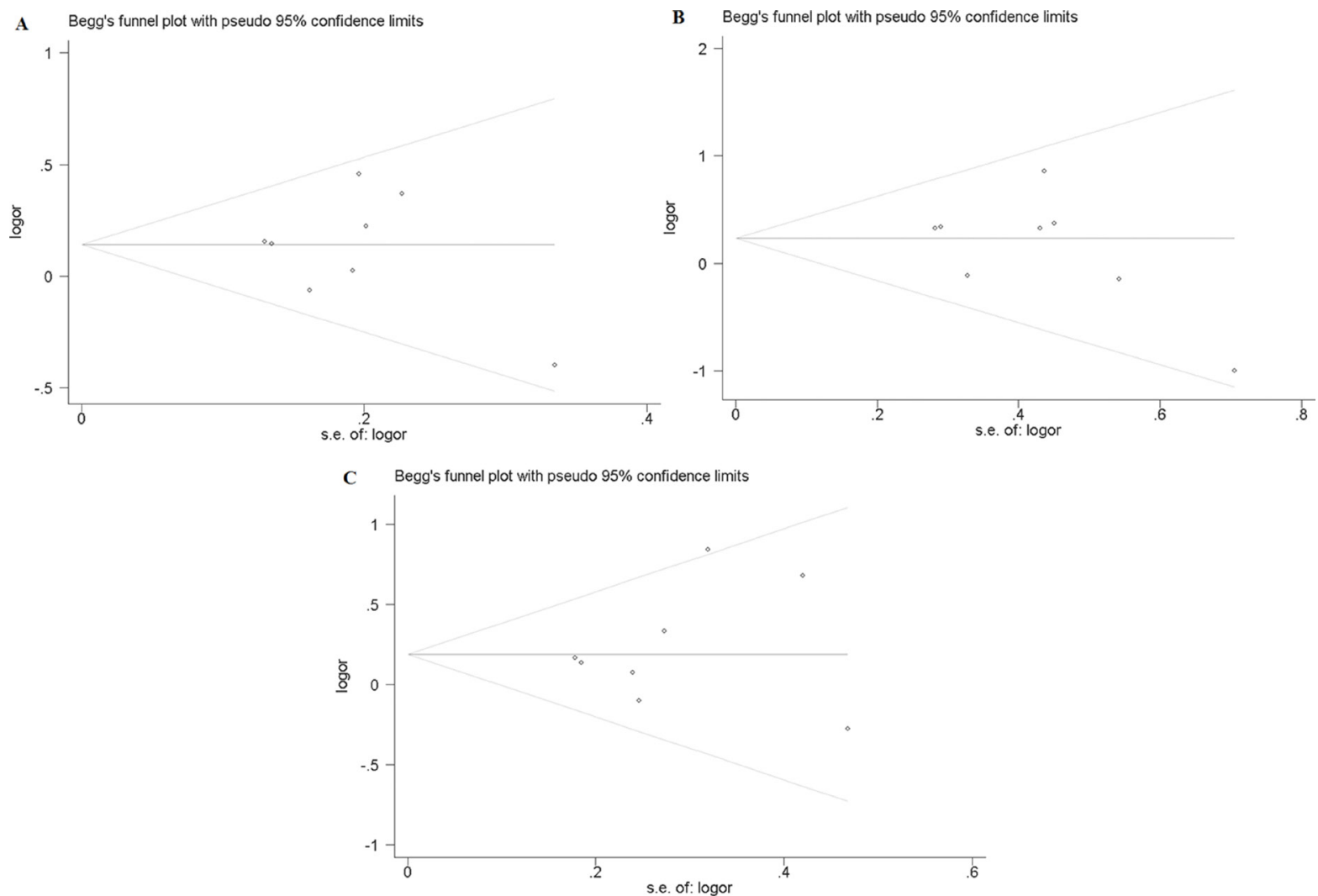

Figure 2: Publication bias of FokI polymorphism. (A) Begg's test for publication bias in allelic model (F vs. f). (B) Begg's test for publication bias in homozygote comparison model (FF vs. ff). (C) Begg's test for publication bias in recessive model (FF vs. Ff $+\mathrm{ff}$ ).
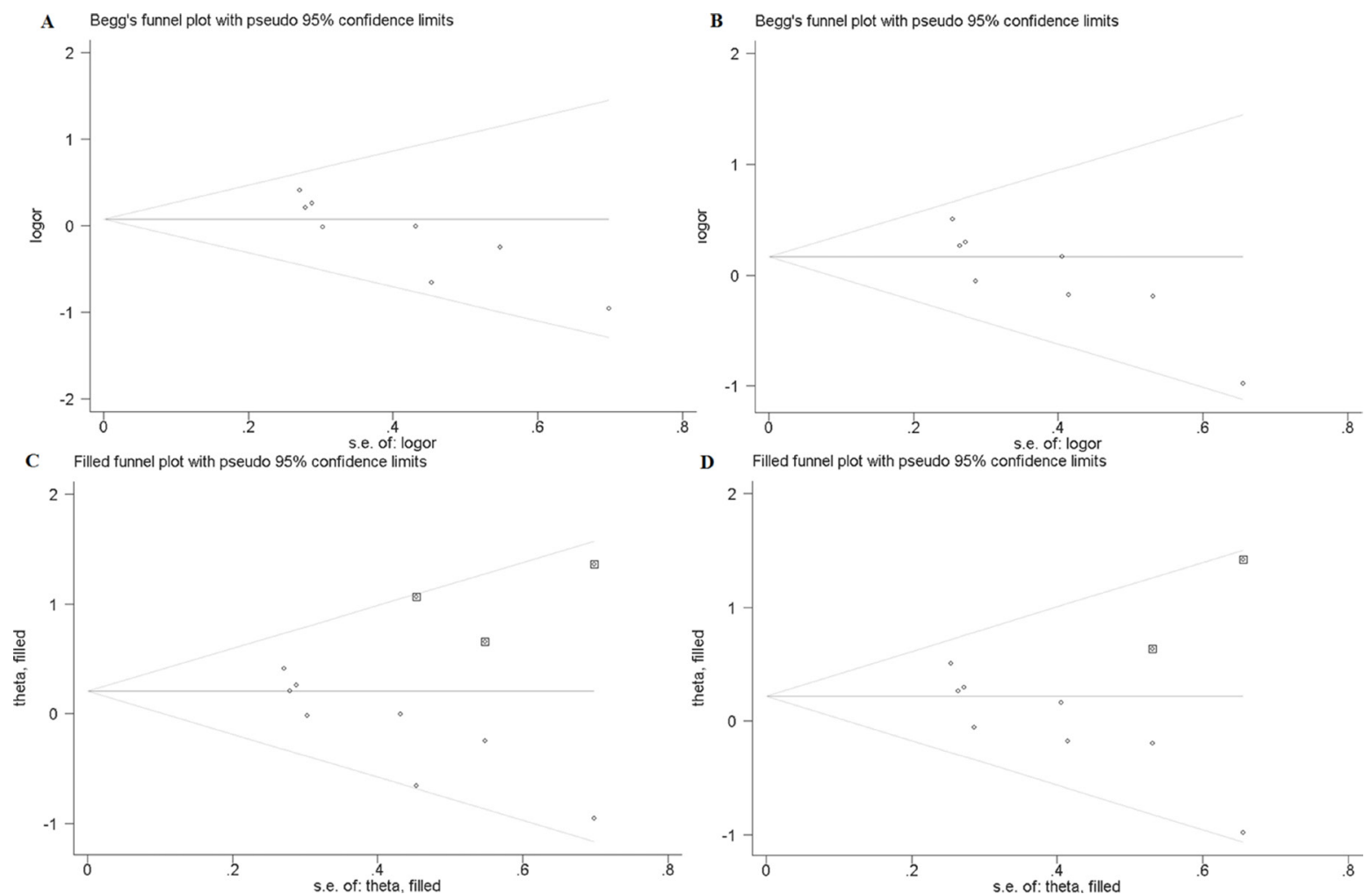

Figure 3: (A) Begg's test for publication bias in heterozygote model (Ff vs. ff). (B) Begg's test for publication bias in dominant model (FF + Ff vs. ff). (C) Trim and Fill for the bias analysis of Ff vs. ff. (D) Trim and Fill for the publication bias analysis of FF + Ff vs. ff. 
The pooled results of fixed-effect model suggest that Ff genotype is not a risk factor for spinal diseases compared with ff carriers $(\mathrm{OR}=1.085,95 \% \mathrm{CI}, 0.851-1.382)$. The $Z$ test of OR has no statistical differences $(Z=0.66$, $p=0.511)$.
As shown in Figure 7, there is no obvious correlation between gene polymorphism and the increased risk of spinal diseases in the dominant model $(\mathrm{FF}+\mathrm{Ff}$ vs. ff: $\mathrm{OR}=1.179,95 \% \mathrm{CI}, 0.938-1.481)$. The results of $\mathrm{Z}$ test also present no significant association $(\mathrm{Z}=1.41$,

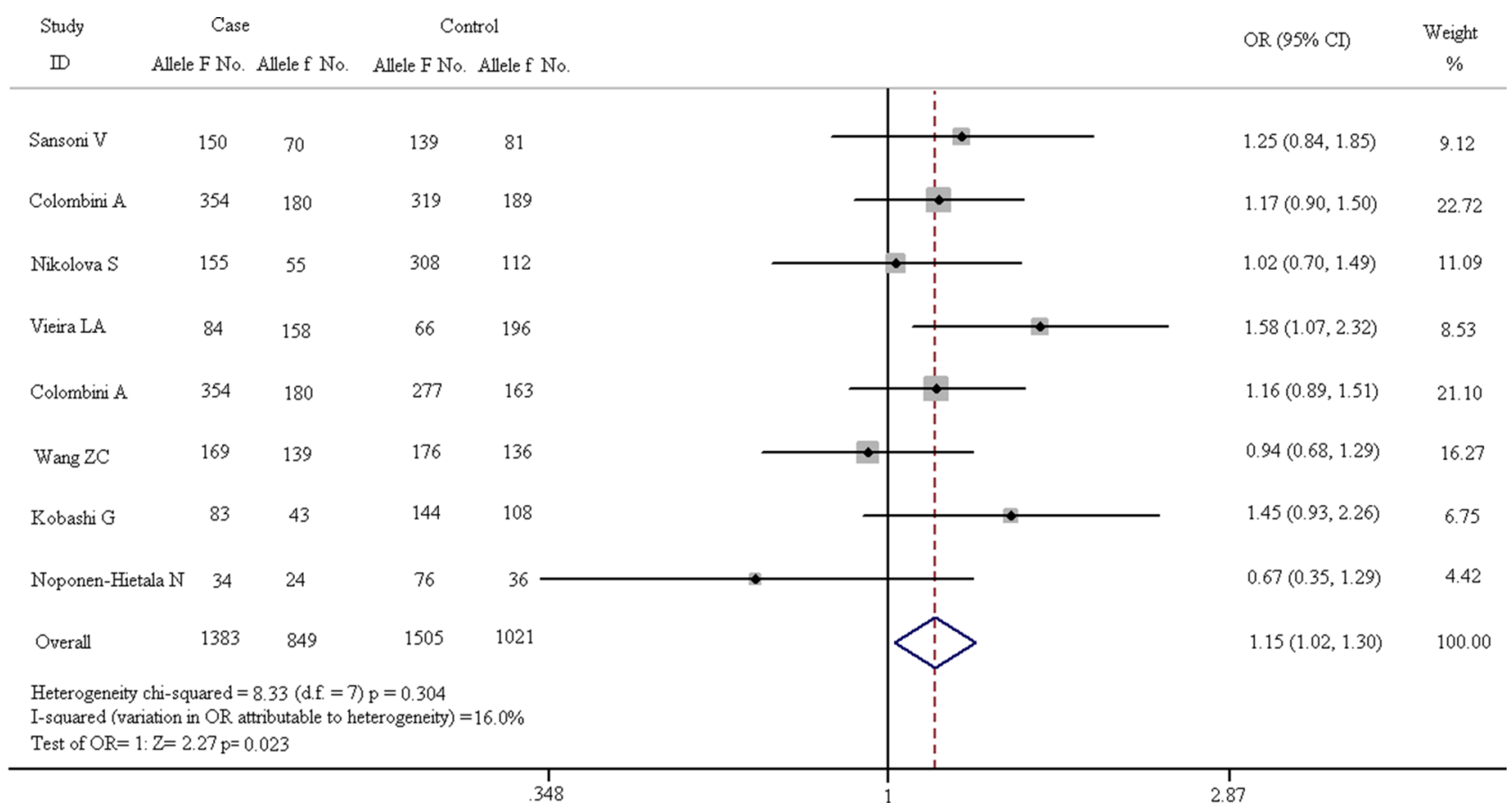

Figure 4: Forest plots of all eligible studies on allele of VDR FokI and the risk of spinal diseases.

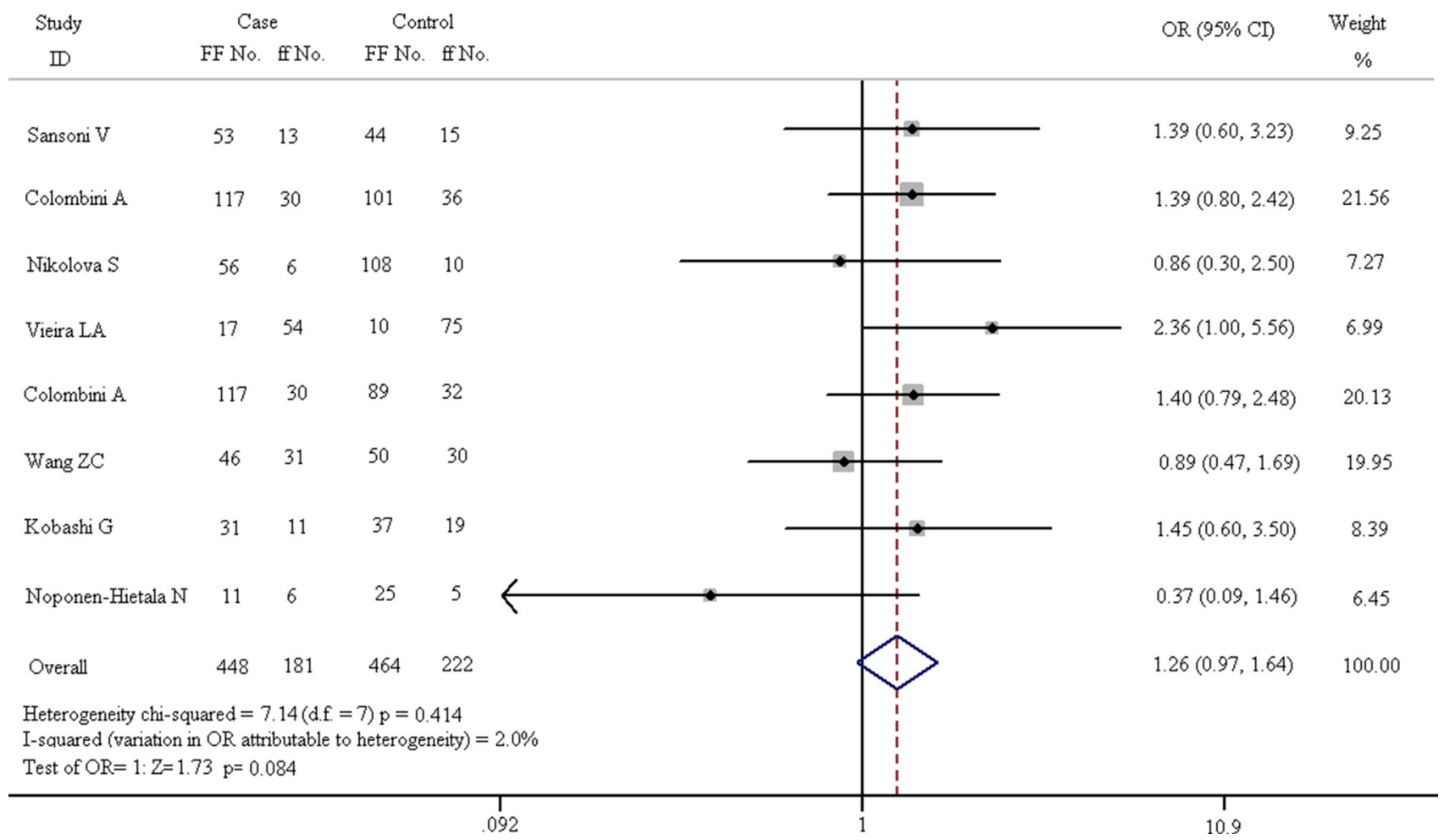

Figure 5: Forest plots of homozygote comparison (FF vs. ff) in all enrolled studies. 
$p=0.158)$. In fixed-effect model, the result of heterogeneity chi-squared test has no significant statistical differences (chi-squared $=6.97, \mathrm{I}^{2}=0.0 \%, p=0.432$ ) . The results of recessive model (FF vs. Ff $+\mathrm{ff}$ ) are shown in Figure 8. In fixed-effect model, the heterogeneity chisquared value is 8.60 and there is no significant difference $\left(\mathrm{I}^{2}=18.6 \%, p=0.282\right)$. The pooled OR is $1.209(95 \% \mathrm{CI}$, $1.017-1.436)$ and $Z$ test shows significant difference $(Z=$ 2.16, $p=0.031)$.

\section{Sensitivity analysis and subgroup analysis}

\section{Sensitivity analysis}

In order to evaluate the influence of each study on the pooled OR, sensitivity analysis is performed and the STATA command "metaninf" is used. One study is expurgated from all eligible articles each time, then the new combined ORs are compared with the original pooled ORs, which are calculated using the data in all the eligible articles. As shown in Figure 9 and Figures 10A-10D, every removed individual article would not significantly affect the overall results, which suggests that the stability of this meta-analysis is high.

\section{Subgroup analysis}

Source of control, quality of literature, population, diagnostic methods and classification of diseases are considered as influential factors for subgroup analysis. Noponen-Hietala N's study is population-based study, while, the quality of the evaluation results is low. Therefore, other 7 hospital-based studies have become a new research subgroup. Furthermore, subgroup analysis is also divided into caucasian group, MRI detection method group and degenerative spine diseases group according to ethnicity, different diagnostic method and classification of disease (Idiopathic scoliosis is excluded). The contrastive analysis results of these subgroups are shown in Table 3.

\section{DISCUSSION}

The onset of spinal diseases is affected by many factors. Behavioral factors, environmental factors and genetic factors are the most thoroughly researched influential factors. Currently, genetic factors turn into research highlights in spinal disease instead of non-genetic factors. A large number of studies indicated that VDR gene plays crucial roles in the etiology of spinal diseases. FokI, BsmI, ApaI and TaqI are the most common types of VDR gene. Various studies have shown that FokI polymorphism is the only one that results in different structure of VDR protein and it is associated with increased risk of spinal diseases [25]. However, other investigations suggest that the FokI polymorphism of VDR is unrelated with the pathogenesis of spinal diseases. The conclusions of these studies are inconsistent. Therefore, we conduct this meta-analysis to reveal the relationship between FokI polymorphism and spinal diseases. In the 8 enrolled studies, the results of 4 articles indicate that allele F and FF genotype of VDR FokI increased the risk of spinal diseases. Meanwhile, the consequences of other 4 studies are opposite. The results of all eligible articles in this meta-analysis illustrate that allele $\mathrm{F}$ is a risk factor for spinal disease with the comparison to allele

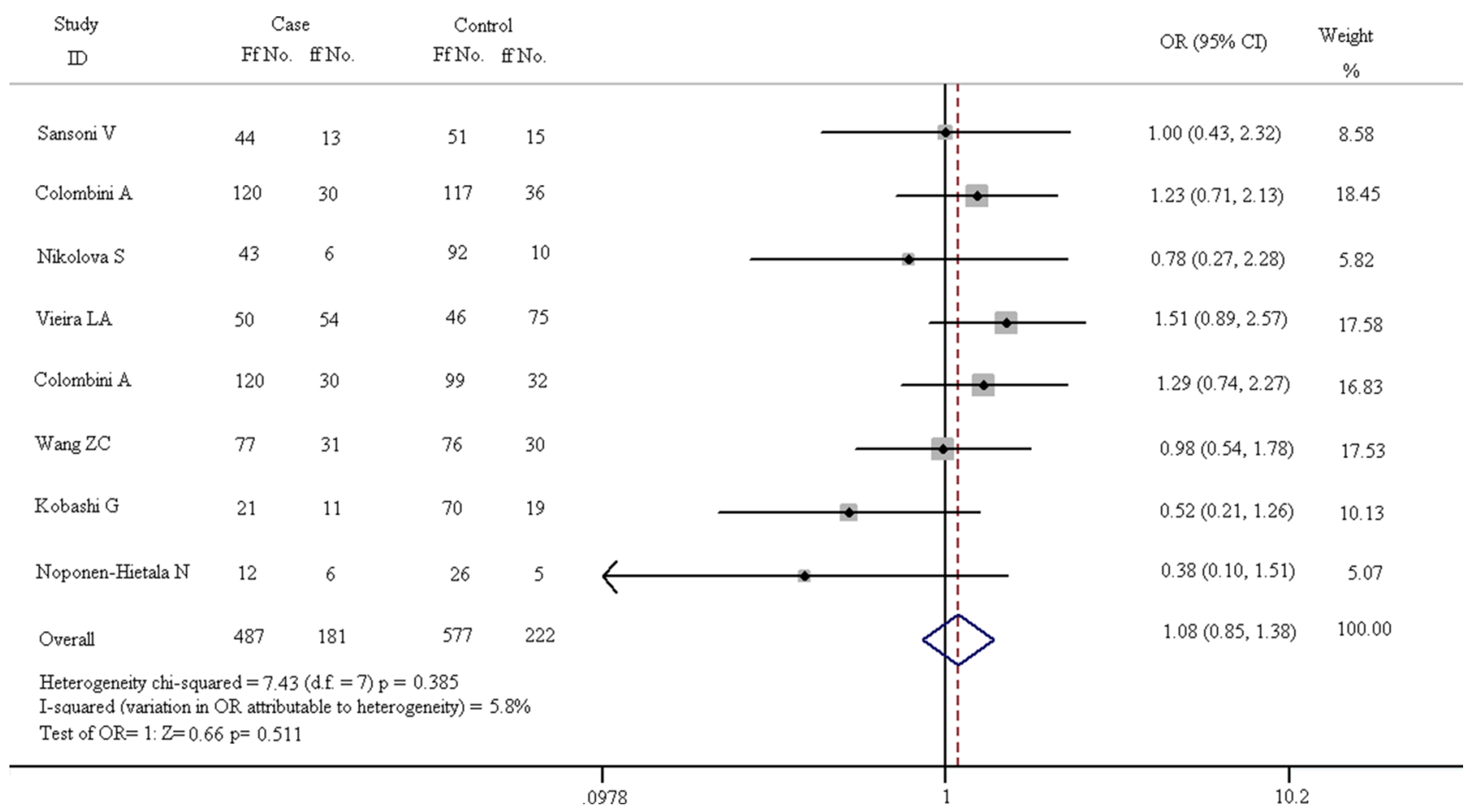

Figure 6: Forest plots of heterozygote comparison (Ff vs. ff) in all eligible articles. 
$\mathrm{f}$ and the difference is significant difference $(p<0.05)$. In the recessive model ( FF vs. Ff $+\mathrm{ff}$ ), the results suggest that FF carriers have a higher risk of spinal diseases compared with the population who carry the recessive gene with statistically significant differences $(p<0.05)$. However, in the genotype analysis of VDR FokI including dominant and (FF + Ff vs.

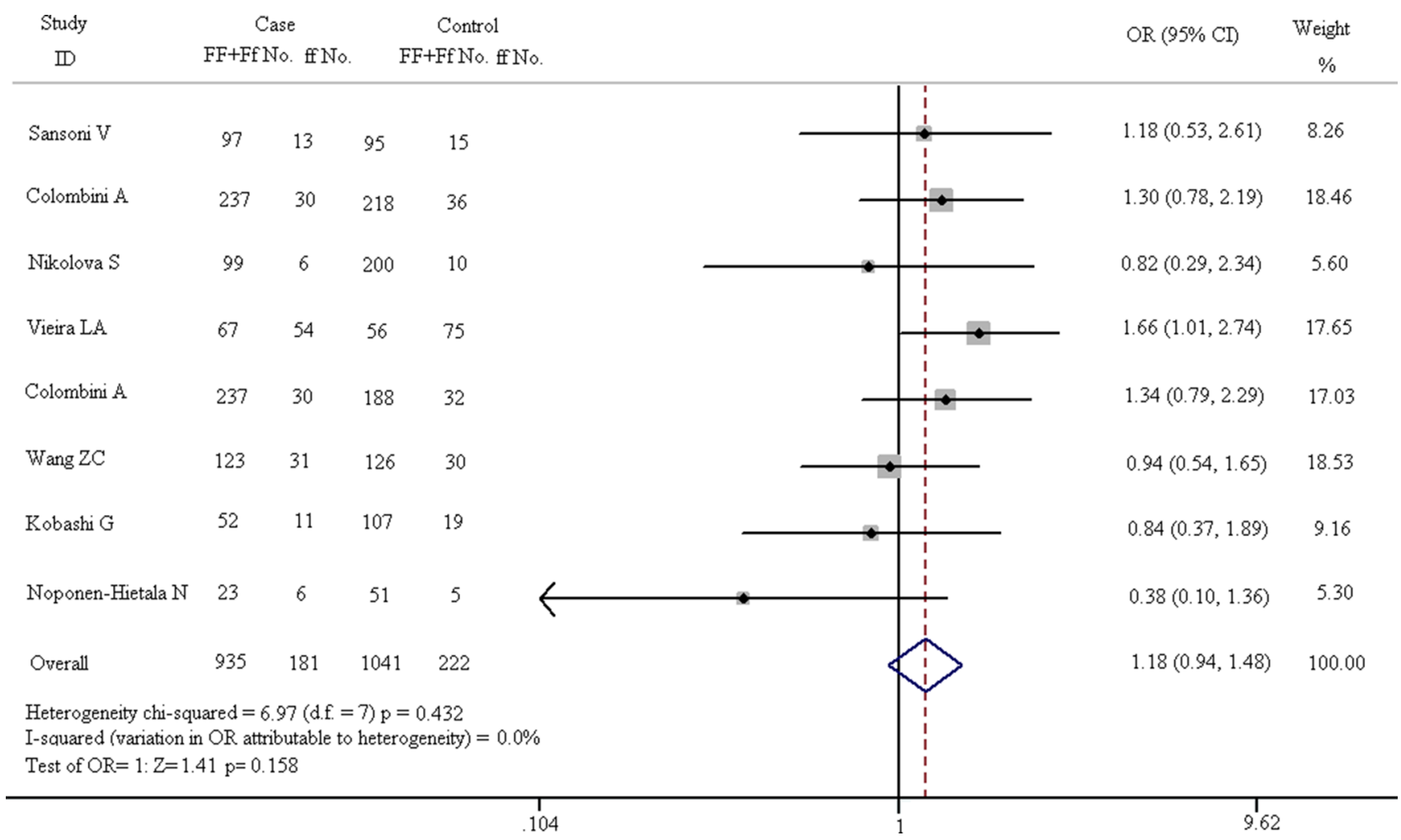

Figure 7: Forest plots in dominant model (FF + Ff vs. ff).

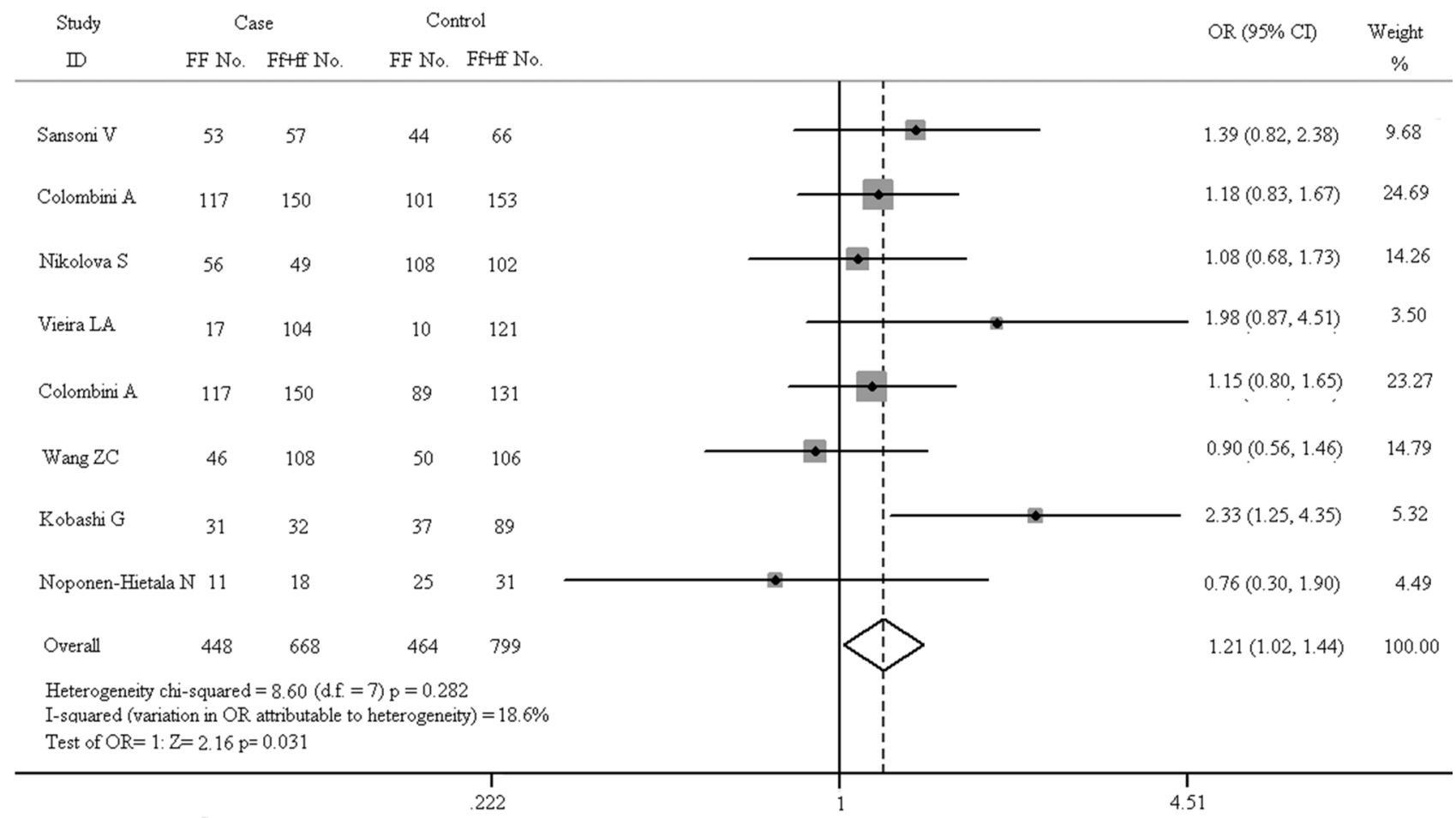

Figure 8: Forest plots in recessive model (FF vs. Ff + ff). 


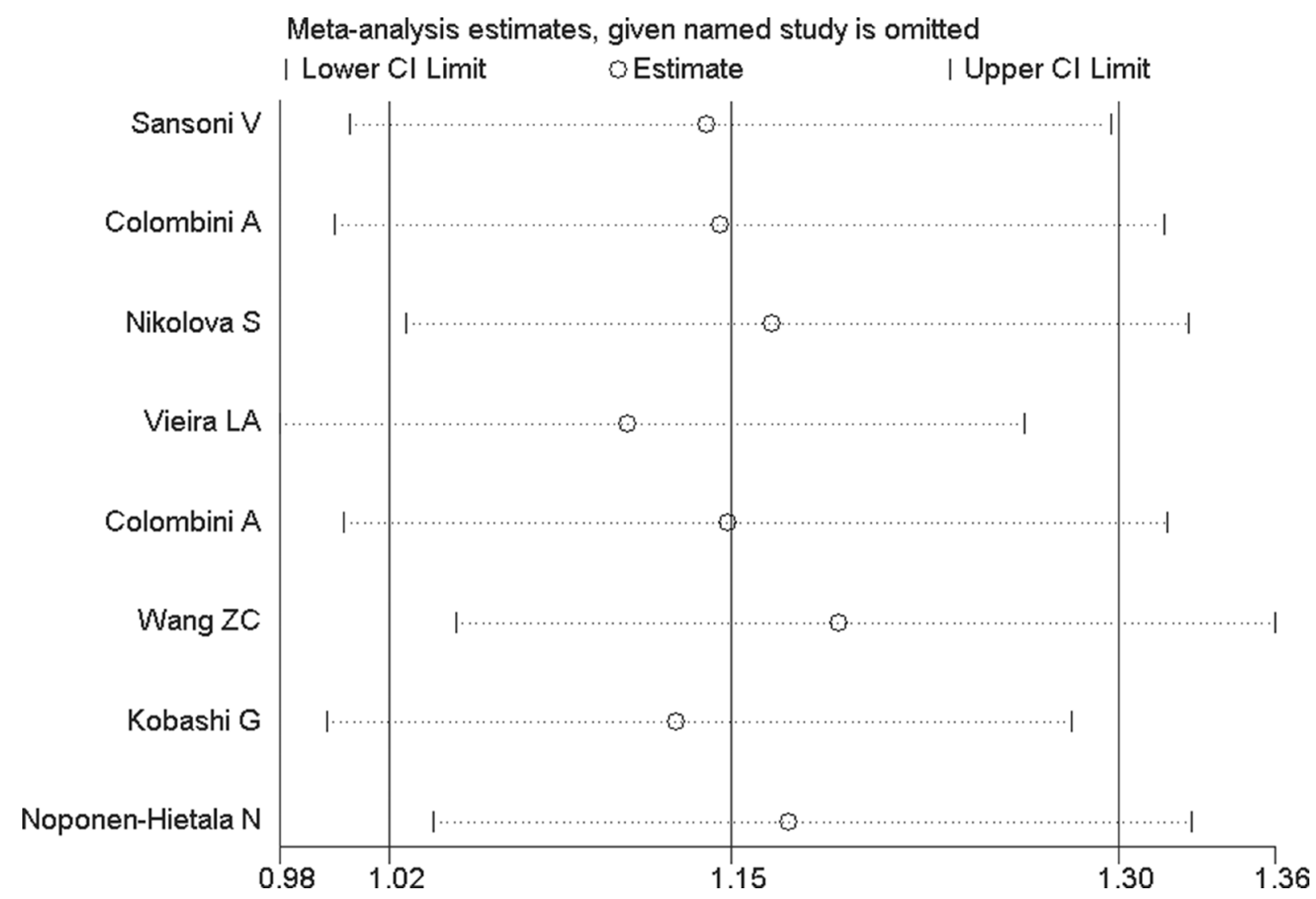

Figure 9: Sensitivity analysis of VDR FokI allelic comparison (F vs. f).
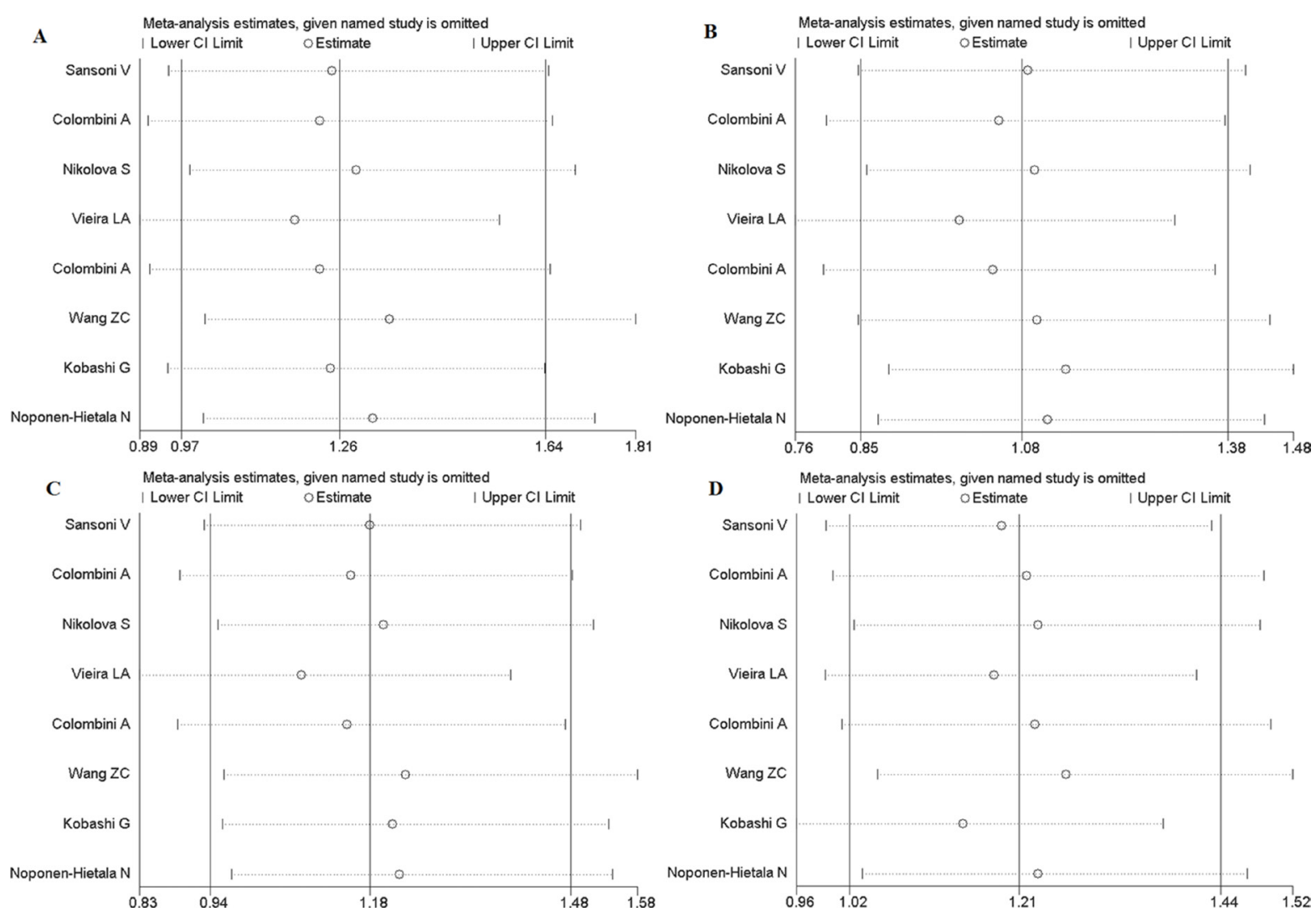

Figure 10: Sensitivity analysis of FokI polymorphism. (A) Homozygote comparison (FF vs. ff). (B) Heterozygote comparison (Ff vs. ff). (C) Dominant model (FF + Ff vs. ff). (D), Recessive models (FF vs. Ff + ff). 
Table 3: Subgroup analysis of VDR FokI polymorphism on spinal diseases

\begin{tabular}{|c|c|c|c|c|c|}
\hline \multirow{2}{*}{ Genetic model } & \multirow{2}{*}{ Analysis model } & \multicolumn{2}{|c|}{ Test of association } & \multicolumn{2}{|c|}{ Test for heterogeneity } \\
\hline & & OR $(95 \% \mathrm{CI})$ & $p$ value & $I^{2}(\%)$ & $p$ value \\
\hline \multicolumn{6}{|c|}{ Hospital-based study } \\
\hline F vs. f & Fixed-effect & $1.174(1.037,1.328)$ & $0.011 *$ & 0 & 0.464 \\
\hline FF vs. ff & Fixed-effect & $1.323(1.011,1.731)$ & $0.041^{*}$ & 0 & 0.682 \\
\hline Ff vs. ff & Fixed-effect & $1.122(0.877,1.436)$ & 0.36 & 0 & 0.522 \\
\hline$F F+F f$ vs. ff & Fixed-effect & $1.224(0.970,1.554)$ & 0.089 & 0 & 0.669 \\
\hline$F F$ vs. $F f+f f$ & Fixed-effect & $1.230(1.032,1.466)$ & $0.021 *$ & 20.7 & 0.271 \\
\hline \multicolumn{6}{|c|}{ Caucasian population } \\
\hline F vs. f & Fixed-effect & $1.17(1.019,1.343)$ & $0.026^{*}$ & 12 & 0.338 \\
\hline FF vs. ff & Fixed-effect & $1.343(0.989,1.824)$ & 0.059 & 13.2 & 0.330 \\
\hline Ff vs. ff & Fixed-effect & $1.189(0.900,1.572)$ & 0.223 & 0 & 0.514 \\
\hline$F F+F f$ vs. ff & Fixed-effect & $1.282(0.985,1.667)$ & 0.065 & 5.9 & 0.379 \\
\hline$F F$ vs. $F f+f f$ & Fixed-effect & $1.190(0.981,1.445)$ & 0.077 & 0 & 0.710 \\
\hline \multicolumn{6}{|c|}{ Diagnostic method (MRI) } \\
\hline F vs. f & Fixed-effect & $1.144(1.000,1.308)$ & $0.049 *$ & 27.9 & 0.225 \\
\hline FF vs. ff & Fixed-effect & $1.277(0.960,1.699)$ & 0.093 & 23.7 & 0.256 \\
\hline Ff vs. ff & Fixed-effect & $1.174(0.906,1.522)$ & 0.225 & 0 & 0.542 \\
\hline$F F+F f$ vs. ff & Fixed-effect & $1.238(0.970,1.581)$ & 0.086 & 12.1 & 0.338 \\
\hline FF vs. $F f+f f$ & Fixed-effect & $1.157(0.953,1.405)$ & 0.140 & 0 & 0.557 \\
\hline \multicolumn{6}{|c|}{ Degenerative spine diseases } \\
\hline F vs. f & Fixed-effect & $1.167(1.026,1.327)$ & $0.018^{*}$ & 24.3 & 0.244 \\
\hline FF vs. ff & Fixed-effect & $1.292(0.985,1.696)$ & 0.064 & 9.4 & 0.357 \\
\hline Ff vs. ff & Fixed-effect & $1.104(0.861,1.415)$ & 0.437 & 15.0 & 0.316 \\
\hline$F F+F f$ vs. ff & Fixed-effect & $1.20(0.949,1.516)$ & 0.127 & 7.6 & 0.370 \\
\hline FF vs. Ff + ff & Fixed-effect & $1.23(1.022,1.480)$ & $0.028 *$ & 28.1 & 0.214 \\
\hline
\end{tabular}

$* \boldsymbol{p}<0.05$

$\mathrm{ff})$, heterozygote comparison (Ff vs. $\mathrm{ff}$ ) and homozygote comparison ( $\mathrm{FF}$ vs. ff), any kind of genotype do not increase the risk of spinal diseases and no statistical difference exists in any estimates $(p>0.05)$. In the eligible literatures, there is one literature with poor quality or different control source. The vast majority of eligible articles are hospitalbased study and only one paper is population-based study. When the individual study is removed, the results of studies with hospital-based controls are slightly different from the results of all enrolled studies. We found that FF genotype carriers are vulnerably threatened by the spinal diseases compared with ff genotype and the population carried the recessive gene, meanwhile, the difference has the statistical significance $(p<0.05)$. Furthermore, subgroup analysis is also divided in to caucasian group, MRI detection method group and degenerative spine diseases group according to ethnicity, different diagnostic method and classification of disease. In the subgroup of caucasian and MRI detection 
method, only the difference in the allelic model (F vs. f) is statistically significant $(p<0.05)$. In degenerative spine diseases group, the results are consistent with that of all enrolled studies.

Our meta-analysis is quite rigorous and has many prominent advantages. First of all, this paper is focused on VDR FokI polymorphism and the risk of spinal diseases. Literatures are chosen from PubMed and Web of science etc., which are the open classic biomedical databases. A reasonable search strategy is designed. Language type and the period covered by the publications are also limited strictly. Secondly, reasonable search strategy, objective quality evaluation, specific inclusion criteria and strict exclusion criteria are conducted to ensure the credibility of this meta-analysis. Finally, suitable statistical methods are used to calculate the results. Sensitivity analysis and stratification analysis are also performed to control the confounding factors.

However, some limitations of this study should not be neglected. The results of the meta-analysis may be influenced by certain limitations. Firstly, pathogenesis of spinal diseases could be affected by non-genetic or genetic factors separately, that is certified by numerous investigations. However, it is worthy to notice that the interaction between genetic factors and non-genetic factors may impact the occurrence of spinal diseases. For example, genetic factors with the interaction of environmental factors increase the risk of spinal diseases. Secondly, confounding variables such as sex, age, smoking and alcohol habits, etc. should be considered accurately and controlled preferably. The data of this meta-analysis are collected from published literatures and it is impossible to eliminate publication bias completely. We can only minimize the effect of publication bias to obtain more reliable results.

\section{MATERIALS AND METHODS}

A detailed literature search strategy is performed using the keywords "Vitamin D receptor (VDR)", "FokI polymorphism", "cervical vertebra pathologies" and "lumbar spine pathologies" to search all the related articles in PubMed, which is a widely used online biomedical database (ultimate search updated on December 13, 2016). We also searched the papers in the frequently-used databases, such as "Web of Science", "Medline", etc. The search strategy consist of the terms "Vitamin D receptor (VDR)", "FokI polymorphism" combined with "cervical vertebra pathologies" and/or "lumbar spine pathologies". Two independent investigators screened the relevant articles using standardized screening guide.

\section{Inclusion criteria}

a. The included studies must be concentrated on the relationship between VDR FokI polymorphism and the diseases of spine. b. Case-control study is the unique methodology in selected articles.

c. Studies with enough data to calculate odds ratios and corresponding 95\% confidence intervals (ORs, 95\% CIs) are included.

d. Cases are diagnosed by diagnostic radiology and clinical diagnosis.

\section{Exclusion criteria}

a. Abstracts, letters, comments, editorials, reviews, single-case reports and family-based studies are excluded.

b. The patients who received chemotherapy, radiotherapy or surgical operation treatment are excluded.

c. The articles with insufficient data or overlapped data are excluded.

\section{Quality assessment}

We evaluate the quality of eligible studies in accordance with an improved 10-point scale, which is the appropriate quality assessment for case-control study $[12,13]$. Quality evaluation parameters and standards of this modified scoring system (range, $0-10$ points) is shown in Table 4, the higher score means the better quality of article. The average score for the eligible studies is 8 points.

\section{Statistical analysis}

This meta-analysis is performed using STATA software (version 12.0, STATA Corp., College Station, TX, USA). Crude Odds ratios (ORs) and corresponding 95\% confidence intervals (CIs) are calculated to assess the strength of association between VDR gene polymorphisms and the susceptibility of spinal diseases. Pooled ORs are calculated using the data of eligible articles in random-effect model (M-H heterogeneity method) or fixed-effect model (Mantel and Haenszel method). $\mathrm{I}^{2}$ index and $p$ value of the chi-squared test are used to inspect the heterogeneity among enrolled literatures. If notable heterogeneity exist $(p<0.05$ and/or $\mathrm{I}^{2}>50 \%$ ), the random-effect model is used to estimate ORs [14, 15]; conversely, the fixed-effect model is performed [16]. ORs are calculated in allele contrastive analysis (F vs. f), homozygote comparison (FF vs. ff), heterozygote comparison (Ff vs. ff), dominant (FF + Ff vs. ff) and recessive model (FF vs. Ff + ff). The $\mathrm{Z}$ test and $p$ value of 0.05 are used to judge whether the differences of OR values have statistical significance. Stratification analysis is decided according to source of controls, ethnicity, different diagnostic method and classification of disease. The chi-square test for HardyWeinberg equilibrium (HWE) is performed and the $p$ value greater than 0.05 is considered to be balanced in control population. Sensitivity analysis is conducted to 
Table 4: Quality evaluation parameters and criterion

\begin{tabular}{|c|c|c|c|}
\hline \multirow{2}{*}{ Parameter } & \multicolumn{3}{|c|}{ Score } \\
\hline & 2 & 1 & $\mathbf{0}$ \\
\hline Sample size & $>100$ & 50-100 & $<50$ \\
\hline Control source & Hospital-based study & Population-based study & Unclear \\
\hline $\begin{array}{l}\text { Matching factors of } \\
\text { case and control }\end{array}$ & $>3$ factors & $1-3$ factors & Unclear \\
\hline Basic information & Adequate & Sectional & Inadequate \\
\hline $\begin{array}{l}\text { Diagnostic method and } \\
\text { genotyping method }\end{array}$ & $\begin{array}{c}\text { Positive radiological } \\
\text { diagnosis and PCR-RFLP }\end{array}$ & $\begin{array}{c}\text { Positive radiological } \\
\text { diagnosis or PCR-RFLP }\end{array}$ & $\begin{array}{l}\text { Not commonly } \\
\text { used method }\end{array}$ \\
\hline
\end{tabular}

PCR-RFLP: polymerase chain reaction-restriction fragment length polymorphism

assess the influence of individual studies. Begg's test is applied to evaluate the publication bias.

\section{Author contributions}

$\mathrm{M} L$ and $\mathrm{X}-\mathrm{Y} \mathrm{H}$ designed the research, did investigations, $\mathrm{M} \mathrm{L}$ : drafted the manuscript, analyze data. M L, X-Y H, Y-J N, G-L Z : reviewed literature, drafted the manuscript, and reviewed the manuscript for final publication.

\section{CONFLICTS OF INTEREST} interest.

The authors declare that they have no competing

\section{REFERENCES}

1. Rubin DI. Epidemiology and risk factors for spine pain. Neurol Clin. 2007; 25:353-371.

2. Toktaş ZO, Ekşi MŞ, Yılmaz B, Demir MK, Özgen S, Kılıç T, Konya D. Association of collagen I, IX and vitamin D receptor gene polymorphisms with radiological severity of intervertebral disc degeneration in Southern European Ancestor. Eur Spine J. 2015; 24:2432-2441.

3. Kinoshita H, Tamaki T, Hashimoto T, Kasagi F. Factors influencing lumbar spine bone mineral density assessment by dual-energy X-ray absorptiometry: comparison with lumbar spinal radiogram. J Orthop Sci. 1998; 3:3-9.

4. O’Neill TW, McCloskey EV, Kanis JA, Bhalla AK, Reeve J, Reid DM, Todd C, Woolf AD, Silman AJ. The distribution, determinants, and clinical correlates of vertebral osteophytosis: a population based survey. J Rheumatol. 1999; 26:842-848.

5. Yuan HY, Tang Y, Liang YX, Lei L, Xiao GB, Wang S, Xia ZL. Matrix metalloproteinase-3 and vitamin d receptor genetic polymorphisms, and their interactions with occupational exposure in lumbar disc degeneration. J Occup Health. 2010; 52:23-30.
6. Sakai Y, Matsuyama Y, Hasegawa Y, Yoshihara H, Nakamura H, Katayama Y, Imagama S, Ito Z, Ishiguro N, Hamajima N. Association of gene polymorphisms with intervertebral disc degeneration and vertebral osteophyte formation. Spine (Phila Pa 1976). 2007; 32:1279-1286.

7. Sambrook PN, MacGregor AJ, Spector TD. Genetic influences on cervical and lumbar disc degeneration: a magnetic resonance imaging study in twins. Arthritis Rheum. 1999; 42:366-372.

8. Eser B, Cora T, Eser O, Kalkan E, Haktanir A, Erdogan MO, Solak M. Association of the polymorphisms of vitamin D receptor and aggrecan genes with degenerative disc disease. Genet Test Mol Biomarkers. 2010; 14:313-317.

9. Pouresmaeili F, Jamshidi J, Azargashb E, Samangouee S. Association between Vitamin D Receptor Gene BsmI Polymorphism and Bone Mineral Density in A Population of 146 Iranian Women. Cell J. 2013; 15:75-82.

10. Cai G, Zhang X, Xin L, Wang L, Wang M, Yang X, Li X, Xia Q, Xu S, Ding C, Pan F. Associations between vitamin $D$ receptor gene polymorphisms and ankylosing spondylitis in Chinese Han population: a case-control study. Osteoporos Int. 2016; 27:2327-2333.

11. Valdivielso JM, Fernandez E. Vitamin D receptor polymorphisms and diseases. Clin Chim Acta. 2006; 371:1-12.

12. Bhutta AT, Cleves MA, Casey PH, Cradock MM, Anand KJ. Cognitive and behavioral outcomes of school-aged children who are born preterm: a meta-analysis. JAMA. 2002, 288:728-737.

13. Liu M, Chen L, Zhou R, Wang J. Association between GSTM1 polymorphism and DNA adduct concentration in the occupational workers exposed to PAHs: a meta-analysis. Gene. 2013; 519:71-76.

14. DerSimonian R, Laird NM. Meta-analysis in clinical trials. Control Clin Trials. 1986; 7:177-188.

15. DerSimonian R, Laird N. Meta-analysis in clinical trials revisited. Contemp Clin Trials. 2015; 45:139-145.

16. Mantel N, Haenszel W. Statistical aspects of the analysis of data from retrospective studies of disease. J Natl Cancer Inst. 1959; 22:719-748. 
17. Sansoni V, Perego S, Colombini A, Banfi G, Brayda-Bruno M, Lombardi G. Interplay between low plasma RANKL, VDRFokI polymorphism in lumbar disc herniation independently from age, body mass, and environmental factors: a casecontrol study in the Italian population. Eur Spine J. 2016; 25:192-199.

18. Colombini A, Brayda-Bruno M, Ferino L, Lombardi G, Maione V, Banfi G, Cauci S. Gender differences in the VDR-FokI polymorphism and conventional non-genetic risk factors in association with lumbar spine pathologies in an Italian case-control study. Int J Mol Sci. 2015; 16: 3722-3739.

19. Nikolova S, Yablanski V, Vlaev E, Stokov L, Savov AS, Kremensky IM. Association study between idiopathic scoliosis and polymorphic variants of VDR, IGF-1, and AMPD1 genes. Genet Res Int. 2015; 2015:852196.

20. Vieira LA, De Marchi PL, Santos AA, Christofolini DM, Barbosa CP, Fonseca FL, Bianco B, Rodrigues LM. Analysis of FokI polymorphism of vitamin D receptor gene in intervertebral disc degeneration. Genet Test Mol Biomarkers. 2014; 18:625-629.

21. Colombini A, Brayda-Bruno M, Lombardi G, Croiset SJ, Vrech V, Maione V, Banfi G, Cauci S. FokI polymorphism in the vitamin D receptor gene (VDR) and its association with lumbar spine pathologies in the Italian population: a case-control study. Plos One. 2014; 9:e97027.
22. Wang ZC, Chen XS, Wang WD, Shi JG, Jia LS, Xu GH, Huang JH, Fan L. The genetic association of vitamin $\mathrm{D}$ receptor polymorphisms and cervical spondylotic myelopathy in Chinese subjects. Clin Chim Acta. 2010; 411:794-797.

23. Kobashi G, Ohta K, Washio M, Okamoto K, Sasaki S, Yokoyama T, Miyake Y, Sakamoto N, Hata A, Tamashiro H, Inaba Y, Tanaka H; Japan Collaborative Epidemiological Study Group for Evaluation of Ossification of the Posterior Longitudinal Ligament of the Spine Risk. FokI variant of vitamin $\mathrm{D}$ receptor gene and factors related to atherosclerosis associated with ossification of the posterior longitudinal ligament of the spine: a multi-hospital casecontrol study. Spine (Phila Pa 1976). 2008; 33:E553-558.

24. Noponen-Hietala N, Kyllönen E, Männikkö M, Ilkko E, Karppinen J, Ott J, Ala-Kokko L. Sequence variations in the collagen IX, XI genes are associated with degenerative lumbar spinal stenosis. Ann Rheum Dis. 2003; 62: $1208-1214$.

25. Zhang HQ, Deng A, Guo CF, Wang YX, Chen LQ, Wang YF, Wu JH, Liu JY. Association between FokI polymorphism in vitamin $\mathrm{D}$ receptor gene and susceptibility to spinal tuberculosis in Chinese Han population. Arch Med Res. 2010; 41:46-49. 